\title{
Software Package for Data Quality Assessment of Optical Electronic Airspace Imagery
}

\author{
Olga V. Grigorieva, Denis V. Zhukov, Andrey V. Markov, Alisher G. Saidov \\ A.F. Mozhaisky Military Space Academy, Saint-Petersburg, Russia \\ alenka12003@mail.ru
}

\begin{abstract}
The paper discusses the software package developed by the authors and designed for automated assessment of data quality indicators of optical-electronic satellite imagery. The program implements the possibility of both a priori and a posteriori determination of the main spatial-frequency and radiometric characteristics of recorded images, which makes it possible to compare (verify) the declared and actual parameters of the imaging equipment. In the first case, the calculation of the predicted image quality is performed by the method based on simulation modeling of all elements of the imaging system, including modeling of the spectral irradiance of the earth's surface and taking into account the transfer characteristics of the atmosphere. In the posteriori assessment, it is used the method that allows us to establish quality indicators in the process of analyzing the contrasting brightness differences present on the image on the border of homogeneous objects.
\end{abstract}

Keywords: image quality indicators, modulation transfer function, resolution, signal-to-noise ratio, imaging system modeling, software package. 


\title{
ПРОГРАММНЫЙ КОМПЛЕКС ОЦЕНКИ КАЧЕСТВА ДАННЫХ ОПТИКО- ЭЛЕКТРОННОЙ КОСМИЧЕСКОЙ СЪЕМКИ
}

\author{
О.В. Григорьева, Д.В. Жуков, А.В. Марков, А.Г. Саидов \\ Военно-космическая академия имени А.Ф. Можайского, Санкт-Петербург, Россия \\ alenka12003@mail.ru
}

В работе рассматривается разработанный авторами программный комплекс, предназначенный для автоматизированного оценивания показателей качества данных оптико-электронной космической съемки. В программе реализована возможность как априорного, так и апостериорного определения основных пространственно-частотных и радиометрических характеристик регистрируемых изображений, что позволяет сравнивать (верифицировать) заявленные и реальные параметры съемочной аппаратуры. В первом случае расчет прогнозируемого качества снимка выполняется по методике, основанной на имитационном моделировании всех звеньев системы формирования изображения, включая моделирование спектральной освещенности земной поверхности и учет передаточных характеристик атмосферы. При апостериорной оценке используется метод, позволяющий установить показатели качества в процессе анализа присутствующих на изображении контрастных яркостных перепадов на границе однородных объектов.

Ключевые слова: показатели качества изображения, функция передачи модуляции, разрешающая способность, отношение сигнал/шум, моделирование системы формирования изображения, программный комплекс.

\section{Введение}

Контроль качества данных, регистрируемых оптико-электронной (ОЭ) съемочной аппаратурой (CA) дистанционного зондирования Земли, имеет важное значение для установления степени пригодности обрабатываемых космических снимков для решения различных прикладных задач, а также для верификации (подтверждения) заявленных параметров СА в процессе ее штатной эксплуатации.

В настоящей работе приведено краткое описание созданного в Военно-космической академии имени А.Ф. Можайского программного комплекса (ПК), предназначенного для определения в автоматизированном режиме количественных оценок показателей качества космических ОЭ изображений. В основу данного комплекса были положены методы математического моделирования систем формирования изображений, разработанные группой под руководством А.Ф. Мелькановича [1].

\section{Общее описание программного комплекса}

Качество ОЭ изображений оценивается информативностью - свойством изображения, характеризующим его способность нести сведения о геометрических и фотометрических характеристиках наблюдаемых объектов. Для количественного описания информативности используются соответствующие показатели качества изображений (ПКИ), которые определяются параметрами используемой СА и условиями наблюдения. Основные ПКИ подразделяются на две группы: пространственно-частотные и радиометрические характеристики (для картографической 
СА используют также координатно-измерительные характеристики). К наиболее важным ПКИ первой группы относятся функция передачи модуляции (ФПМ), разрешающая способность и линейное разрешение на местности (ЛРМ). Радиометрические свойства СА определяют градационная характеристика и отношение сигнал/шум $[1,2]$.

Качество изображения оценивается двумя способами:

- анализом параметров СА, предназначенной для получения изображения (априорная оценка или прогнозирование качества ожидаемого изображения);

- анализом характеристик самого изображения, полученного СА в реальных условиях наблюдения с учетом влияния атмосферы и особенностей сцены (апостериорная оценка качества).

В рассматриваемом ПК реализованы оба указанных способа определения ПКИ, что является важным при необходимости сравнения заявленных и реальных параметров СА. Соответственно, в состав ПК входят два основных программно-алгоритмических модуля априорного и апостериорного оценивания качества изображений, а также модуль поддержки информационной базы данных (БД), который предназначен для автоматизированного управления, хранения и поиска информации, необходимой для функционирования ПК.

\section{Модуль априорного оценивания ПКИ}

Модуль априорного оценивания ПКИ выполняет последовательный расчет следующих показателей: ФПМ, отношения сигнал/шум, разрешающей способности и ЛРМ. Блок-схема алгоритма функционирования модуля приведена на рисунке 1.

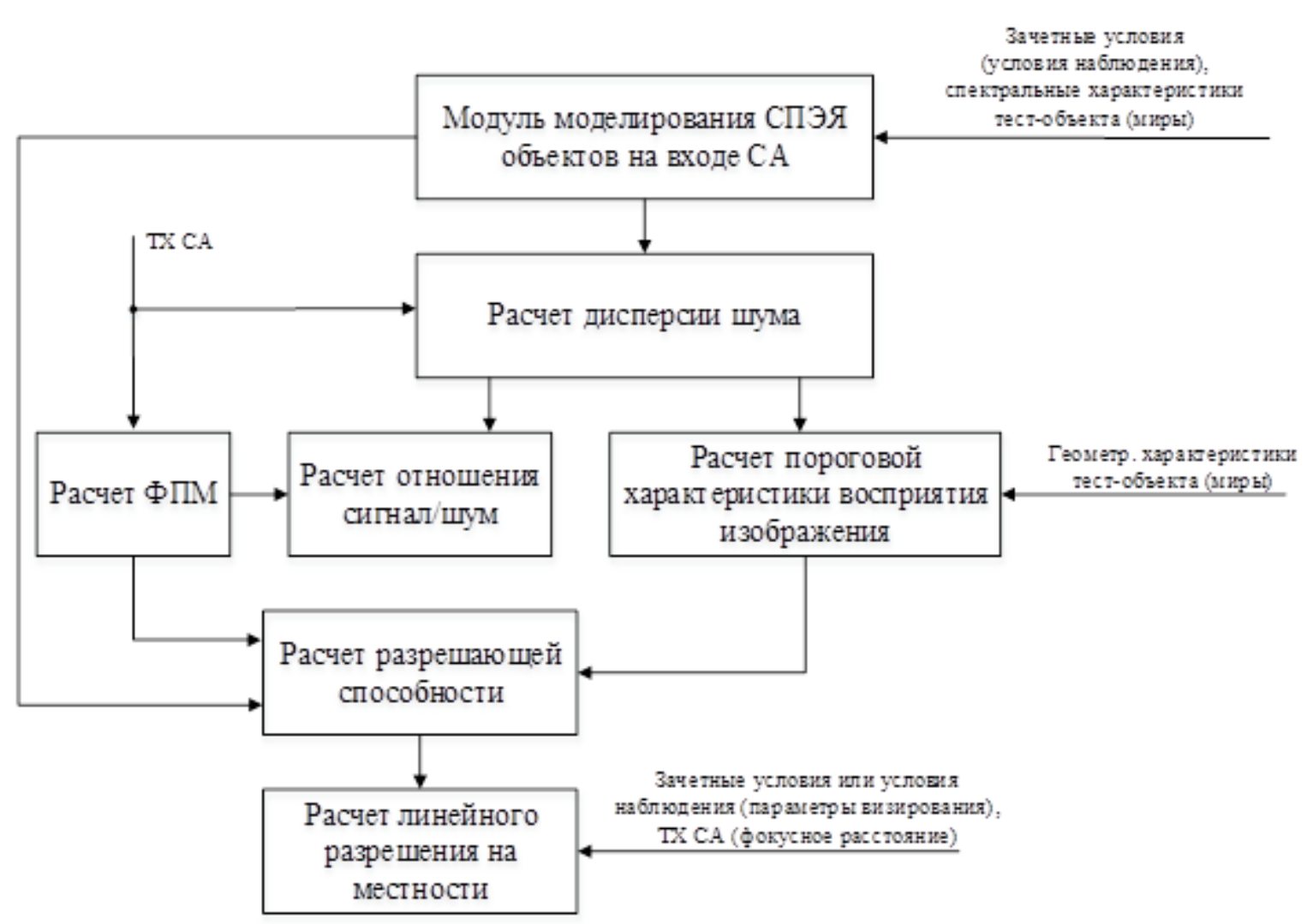

Рис. 1. Блок-схема алгоритма функционирования модуля априорного оценивания ПКИ

Реализованный в ПК алгоритм вычисления прогнозируемых ПКИ основан на имитационной математической модели системы формирования изображения [1], которая позволяет определять значения: 
- облученности сцены и спектральной плотности энергетической яркости (СПЭЯ) наблюдаемых объектов на входном зрачке СА;

- ФПМ оптической системы, сдвига изображения и приемника лучистой энергии и результирующей ФПМ СА;

- пороговой характеристики восприятия изображения, играющей роль математической модели дешифровщика;

- разрешающей способности и ЛРМ.

В методике прогнозирования отношения сигнал/шум учитываются собственный (темновой) шум приемных элементов, шум подстилающей поверхности, шум квантования, шум остаточной неравномерности чувствительности и шум системы регистрации.

Априорная оценка может производиться как с целью анализа соответствия СА предъявляемым к ней тактико-техническим требованиям, так и в интересах прогноза показателей качества СА в условиях реальной эксплуатации (применительно к наблюдению конкретных объектов интереса в различных условиях). В первом случае исходные данные для расчетов (параметры зачетных условий наблюдения, параметры тест-объекта (штриховой миры)) импортируются из БД. Для второго варианта предусмотрена возможность произвольного задания значений геометрических и спектральных характеристик интересующих объектов и фонов, а также параметров условий наблюдения (дата, время, место съемки, углы визирования, метеообстановка). Технические характеристики (ТX) СА также могут извлекаться из БД или задаваться оператором. Пример диалогового окна с результатами априорных расчетов приведен на рисунке 2.

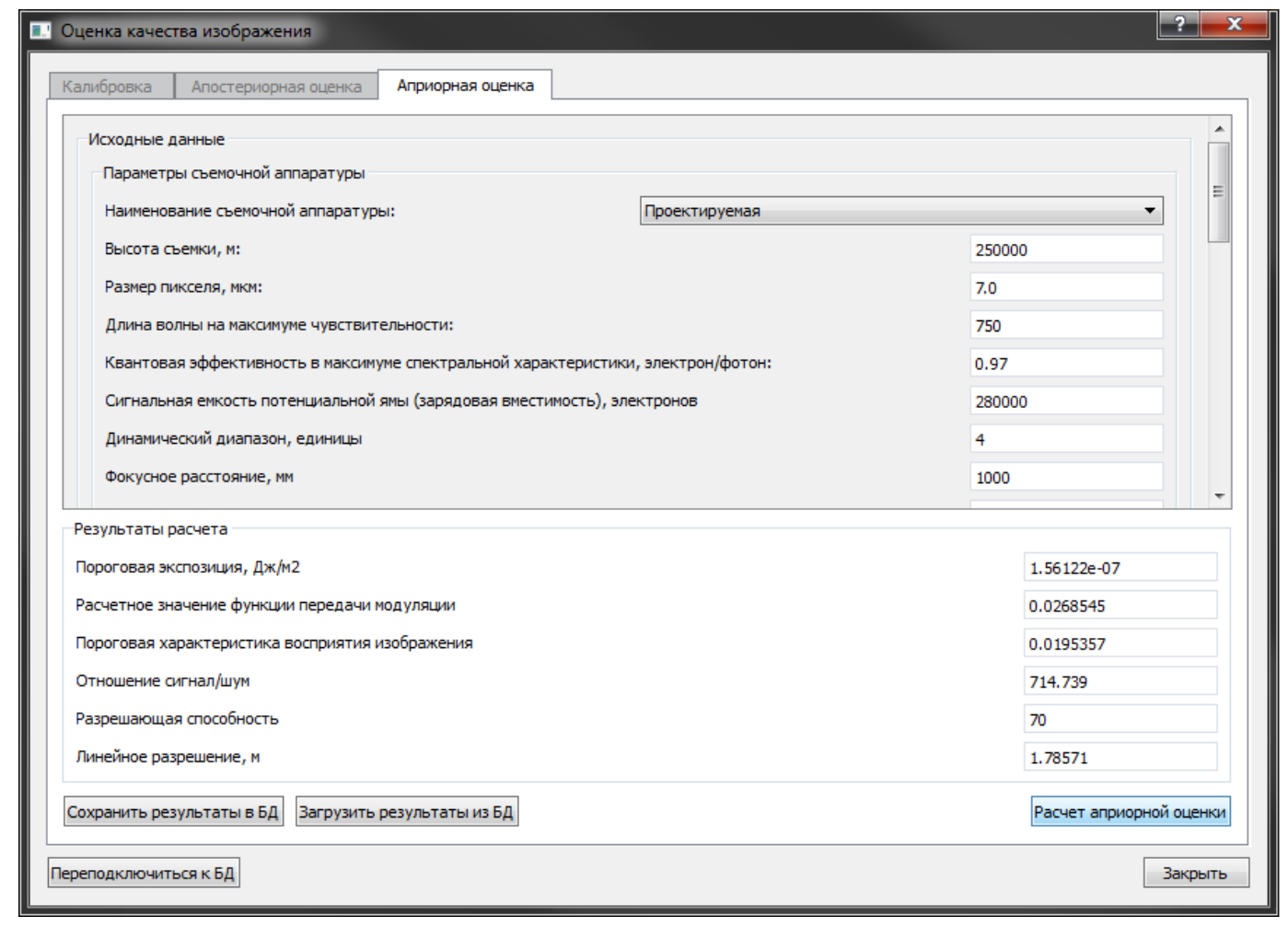

Рис. 2. Результаты расчета априорных оценок ПКИ 


\section{Модуль апостериорного оценивания ПКИ}

Алгоритм работы модуля апостериорного оценивания ПКИ зависит от вида объектов, которые принимаются в качестве тестовых на оцениваемых изображениях, и от наличия данных наземных спектрометрических измерений этих объектов.

В идеальном случае снимок должен содержать изображения специальных мир. Одновременно с космической съемкой должны проводиться наземные спектрометрические и метеорологические измерения. При таких условиях дешифровщик определяет разрешающую способность методом визуального анализа изображения миры [3]. При отсутствии специальных тест-объектов применяется алгоритм обработки, блок-схема которого показана на рисунке 3.

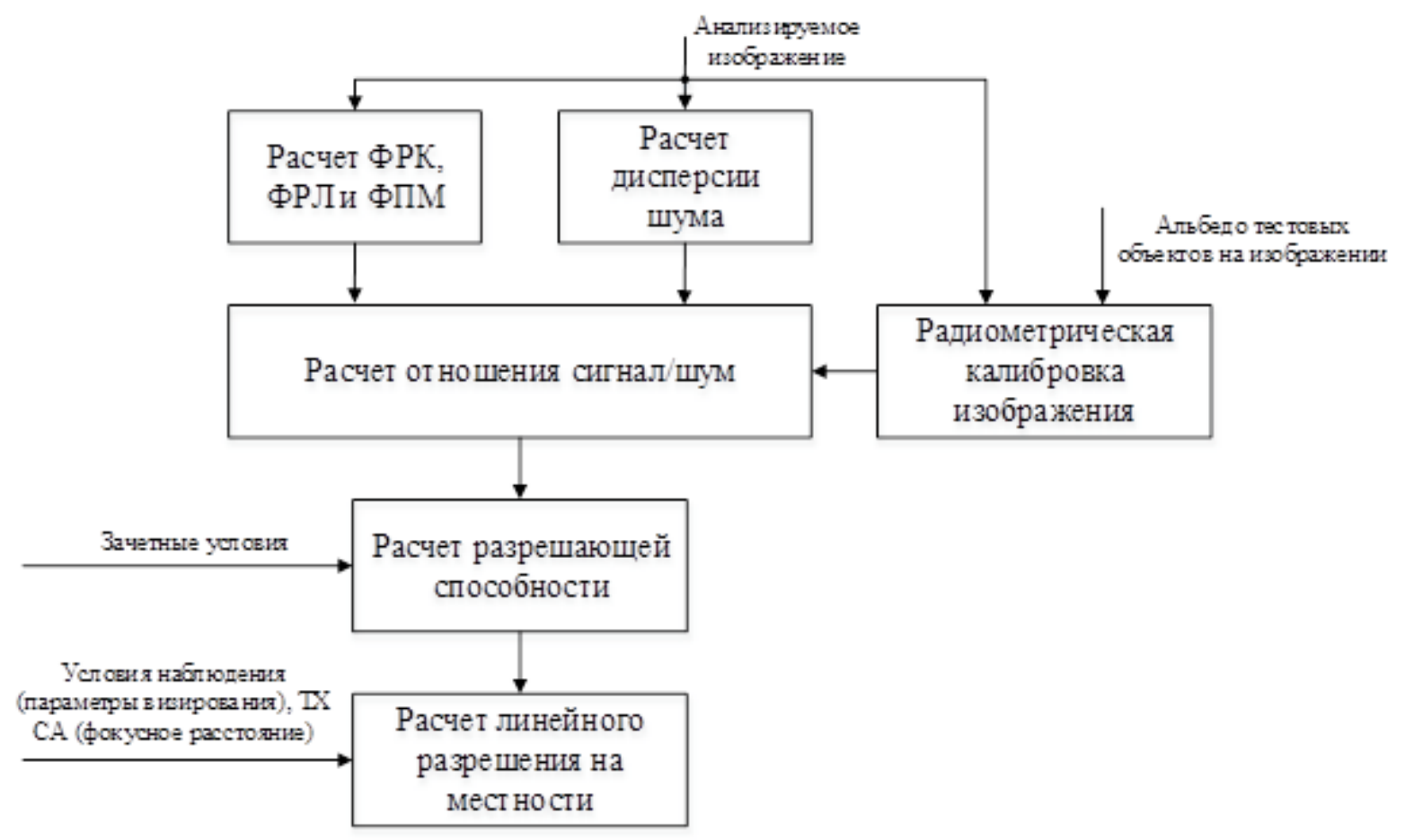

Рис. 3. Блок-схема алгоритма функционирования модуля апостериорного оценивания ПКИ

Первоначально производится радиометрическая калибровка (рассчитывается градационная характеристика) изображения по тестовым объектам с известными отражательными свойствами. При этом необходимо использование результатов синхронных (или квазисинхронных) наземных спектрометрических измерений или, при их отсутствии, задание значений коэффициентов спектральной яркости (альбедо для панхроматической СА) тестовых объектов из БД. В последнем случае оператор должен в качестве тестовых выбирать поверхности со стабильными и маловариативными спектральными характеристиками (бетон, песок, вода и др.).

Следующий этап обработки включает выбор на изображении приблизительно однородных поверхностей, средний уровень сигнала для которых близок среднеарифметическому значению сигналов от полосы и фона условной миры, величины которых рассчитываются автоматически. По среднеквадратическому отклонению сигналов для этих полигонов производится оценка дисперсии шума на изображении. После этого оператор на изображении выбирает в качестве тестовых смежные объекты, на границе которых наблюдается значительный перепад яркости, и обозначает линию, пересекающую эту границу (рисунок 4). После этого автоматически производится последовательный расчет следующих ПКИ: функции рассеяния края (ФРК), функции рассеяния линии (ФРЛ), ФПМ, отношения сигнал/шум, разрешающей способности и ЛРМ. Результаты вычислений отображаются в диалоговом окне (рисунок 5). 


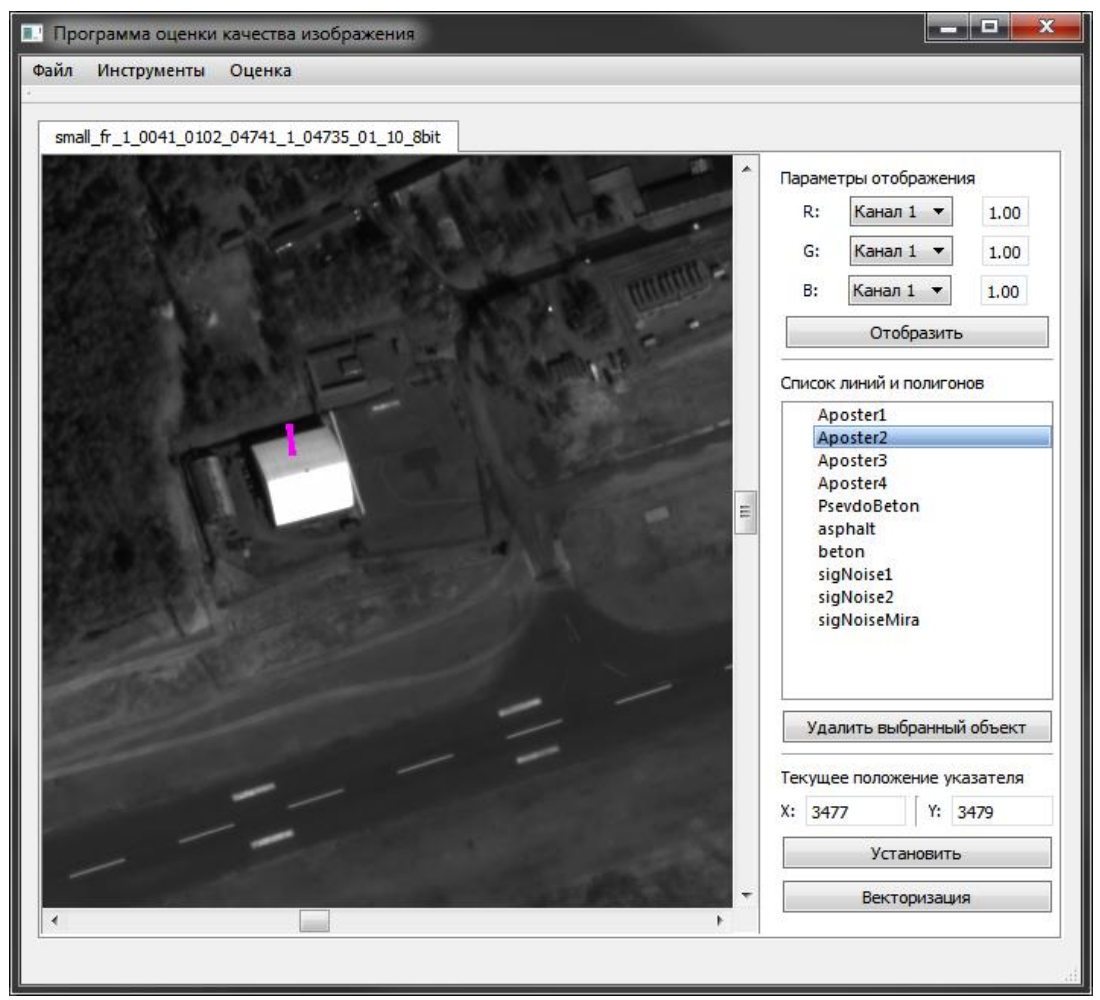

Рис. 4. Обозначение на изображении линии, пересекающей границу объектов с резким яркостным перепадом

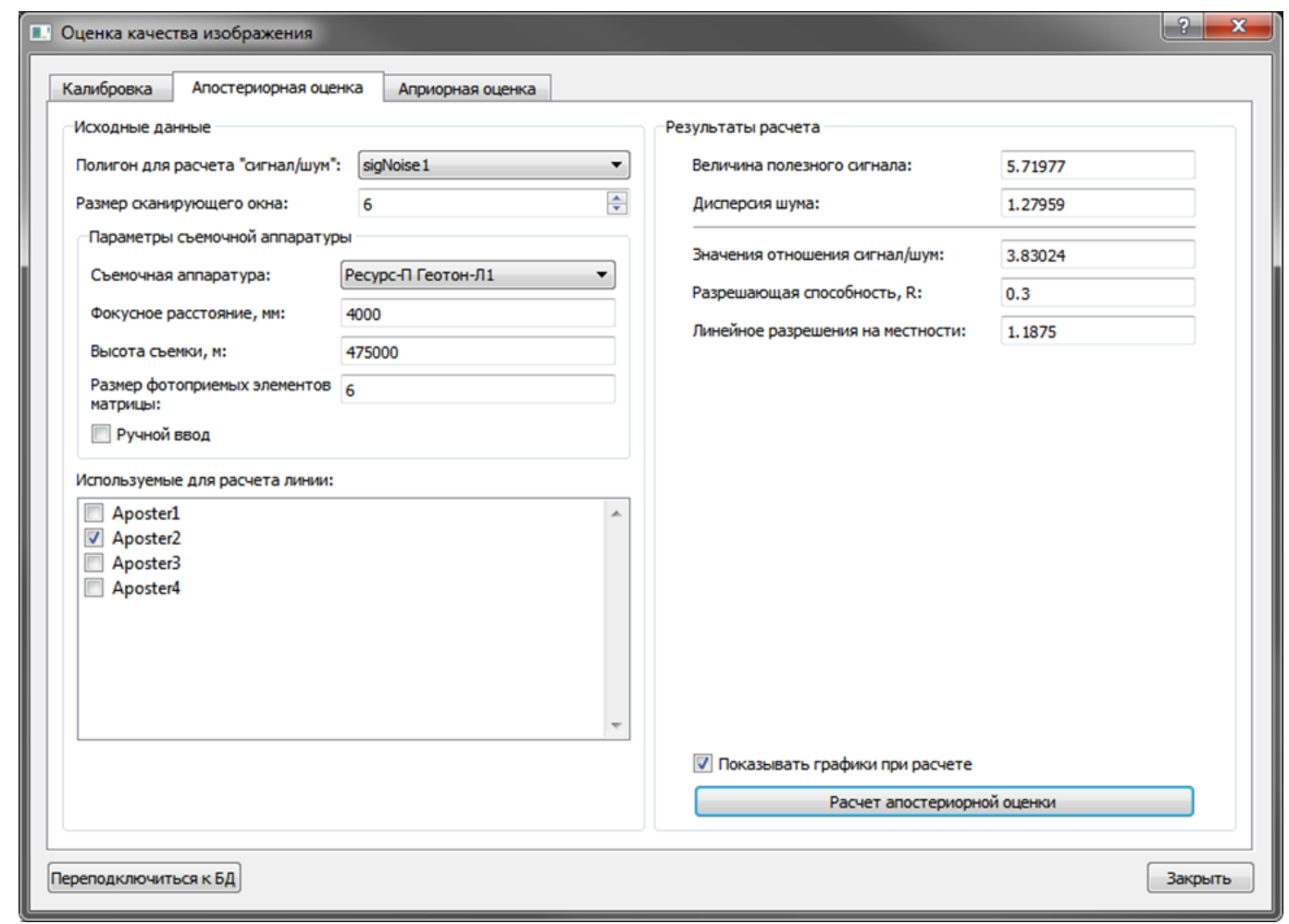

Рис. 5. Результаты расчета апостериорных оценок ПКИ 


\section{References}

[1] Zuev, A. L., Markov, A. V., Milenovic A. F., Kondratenko E. A., Method of justification of parameters of equipment and shooting modes when the analog modeling of the standard equipment of remote sensing of the Earth. In the scientific and technical collection of works of the 2 nd research technical center of the 4th Central research Institute of the Department of Defense of Russian Federation, issue 5, St. Petersburg: A F Mozhaisky Military Space Academy, 2003, pp. 126-133.

[2] Eremeev V.V., Modern technologies of earth remote sensing data processing, Moscow: Fizmatlit, 2015. 460 p.

[3] Bocharnikov A. I., Zhilichkin A.G., Kovalenko V. P., Kondratov A. V., Tikhonychev V.V., Khudiakov A. V. Techniques for Characterization of Remote Sensing Spacecraft Targeted Equipment, Rocket-Space Device Engineering and Information Systems, 2015, Vol. 2, Iss. 2, pp. 18-31. 